\title{
Exploring Haptic Feedback in Exergames
}

\author{
Tadeusz Stach and T.C. Nicholas Graham \\ School of Computing, Queen's University \\ Kingston, ON, Canada \\ \{tstach, graham\}@ics.queensu.ca
}

\begin{abstract}
Exergames combine entertainment and exercise in an effort to encourage people to be more physically active. Although exergames require active input, interactions are less physical than those experienced in real-world exercise. Interactions can feel artificial, limiting the captivating experience exergames aim to provide. To address this problem, haptics have been proposed as a means of providing additional feedback to players through the sense of touch. However, there is very little empirical evidence supporting the benefits of haptics in exergames. To address this, we have identified and evaluated three ways in which haptic feedback can enhance exergames: by helping to balance group exercise among people of different fitness levels, by guiding players toward safe and healthy interaction, and by increasing peoples' sense of virtual presence in exergames. We present three novel exergames incorporating haptic feedback, and report on experiments investigating their success. We find that haptics which are consistent with actions displayed on-screen increase immersion and improve enjoyment. However, we discover pitfalls when using haptics to represent phenomena that do not have a physical basis. These results allow us to present a set of design issues for haptic feedback in exergames.
\end{abstract}

Keywords: Exergames, haptics, force-feedback, exercise video games, exertion interfaces, active games.

\section{Introduction}

Exergames combine video games with exercise in an effort to encourage physical activity. Popular commercial exergames include EA Sports Active, Nintendo's Wii Sports and the Fisher Price Smart Cycle. Exercise inherently connects people to the physical world: people feel their feet impact the ground when running, fight to control the vibrations of their bat when they hit a baseball, and strain when cycling uphill. Traditional video games rely primarily on sight and sound to convey information to players. With exergames, haptic feedback is crucial to activating the tactile sensations that are so strong a part of physical exercise. Although haptic feedback is found in some existing video games, it has received little attention compared to the visual and audio interaction channels, and there has been to-date no systematic treatment of its role in exergaming. 
In this paper we help to address this gap by investigating how haptic feedback can be used to improve three aspects of exergaming:

- Balancing: A major advantage of exergames over traditional physical activity is that games can mediate the effort players must expend, allowing people of disparate physical abilities to play together. We demonstrate how haptics can aid in creating effective and enjoyable balancing in multiplayer exergames.

- Safe and Healthy Interaction: Another significant advantage of exergames is that they can guide players toward a level of exertion that is both safe and beneficial to their health. We demonstrate how haptic feedback can be integrated into gameplay to provide players with subtle cues as to when exertion limits have been reached.

- Presence: The key promise of exergames is that players' enjoyment of physical activity can be enhanced by presenting it through an immersive virtual world. Players' sense of presence is achieved through consistent visual and auditory feedback. We demonstrate that haptics can significantly increase players' feeling of presence within a virtual world.

To illustrate these applications of haptics, we have created three novel exergames. In the following sections, we explore the design of haptic feedback within these games, and report on experiments investigating their success. We found both positive and negative results for haptics. For example, haptics works well when reporting a physical phenomenon in the virtual world, and less well when tied to abstract properties that cannot be directly observed. We conclude by summarizing our lessons learned from exploring haptic feedback in exergames.

\section{Background}

This work builds on and combines existing research in exergaming and haptics. Exergames, a form of "exertion interface" [21], aim to encourage physical activity by combining video games and exercise. Commercial exergaming platforms include the Nintendo Wii, Konami Dance Dance Revolution (DDR), Fisher-Price Smart Cycle, as well as the recently released Sony PlayStation Move and Microsoft Kinect. Numerous academic exergames have also been developed, such as Breakout for Two [21], Push'N'Pull [21], Frozen Treasure Hunter [33], and Swan Boat [1].

Most existing exergames provide at best limited physical output in response to actions occurring in the virtual world. Mueller et al. suggest that in order for exergames to simulate real-world activity, they must include force feedback [21]. For instance, in the Breakout for Two game, players kick soccer balls at a projected wall display to break virtual bricks [21]. This configuration allows the player to feel her foot making contact with a ball. In Remote Impact, players feel the moment of impact when punching and kicking a projected image of their opponent [20]. However, these systems are based on augmented reality and offer no force feedback in response to action occurring in a virtual world. 
The majority of exergaming systems are based on virtual reality. For instance, in Heart Burn, players pedal an exercise bike in order to race their truck along a virtual track [28]. When racing a real car, a driver feels vibrations from rough terrain and forces from rapid turns at high velocity. In Heart Burn, these are represented visually through motion of the truck, but are not transmitted as physical sensations to the player. Similarly, in Swan Boat, players run on a treadmill and use arm motions to control their on-screen boat, but players receive no physical feedback when they collide with virtual objects [1]. As exergames require players to physically exert themselves, this lack of physical response to their actions is keenly felt.

Haptic feedback conveys information to people via applied forces and/or vibrations. Haptic feedback may be tactile or kinesthetic [24]. Tactile feedback provides a sense of touch (e.g., texture and vibration), while kinesthetic feedback leads the user to perceive force (e.g., weight and resistance). Haptics have been used to improve interaction in a variety of contexts. For instance, haptic awareness in distributed groupware systems has been found to improve task performance and peoples' sense of virtual presence $[3,25]$, and haptic warning systems integrated into automobile steering wheels have been found to decrease peoples' reaction times [29].

Gamepads and joysticks often include hardware mechanisms to provide haptic feedback [7]. These have limited functionality and so can express only the most basic feedback. Gamepads typically support a vibration mode that can be turned on or off; "force feedback" joysticks dynamically adjust the resistance felt when moving the joystick. Most commercial exergaming equipment provides little or no haptic feedback; e.g., the tension setting on the PCGamerBike Mini is not under programmable control, and the Wii Remote provides a limited vibration mode similar to traditional gamepads.

Haptic feedback has nevertheless been explored in some exergames. In Airkanoid, players swing hand-held paddles to hit virtual balls [9]; the player's paddle vibrates when it hits an on-screen ball. The Push'N'Pull exergame uses a Powergrid Fitness Killowatt controller to provide resistance when players interact with virtual items [21]. Virku allows players to explore a virtual environment by pedaling on an exercise bike [17]. Tension on the bike pedals increases when a player climbs a virtual slope, and decreases as the player descends.

There has been to-date little evaluation of the effectiveness of haptic feedback in exergames. One exception is Morelli and colleagues' work on games for the visually impaired. The VI-Tennis exergame provides audio and tactile cues to notify players when to swing a Wii Remote to hit a virtual ball [18]. Haptic feedback was found to improve the in-game performance of visually-impaired players, and was preferred to audio feedback alone.

\section{Applying Haptic Feedback to Exergames}

Exercise brings people into intimate sensory contact with the physical world around them. A cyclist riding down a hill feels the vibration of the road in her hands, the sideto-side movement of the bike in her core muscles, and the wind in her face. It is important for computer-mediated exercise to preserve this physicality of real-world 
activity in order to provide an engaging and immersive experience. Although it is widely assumed that haptics addresses this issue, to-date there has been surprisingly little research to validate this assumption.

We propose that haptic feedback can help to address three important areas of exergame design: balancing of group exercise so that people of different physical abilities can play together, guidance of players to safe and healthy levels of interaction, and provision of an immersive experience to increase the enjoyment of physical activity. These three areas capture the fundamental promise of exergames: that computer-mediation can overcome the significant barrier in traditional exercise of not having people to work out with [13], that playing can be beneficial (e.g., meeting recommended levels for physical activity), and that game tasks can absorb players' focus and distract them from physical exertion [31].

In this section, we discuss how haptics can contribute to these three design questions, and present three novel exergames to illustrate example design solutions. In the following section, we present the results of a study showing the effectiveness and pitfalls of adding haptics to exergames.

\subsection{Balancing Group Exercise}

Grouping has been shown to be an important motivating factor in exercise [4]. However, it can be difficult for people of disparate abilities to exercise together [2]. Computer mediation can help balance exercise so less fit people can maintain a sense of competitiveness. We argue that haptics are a useful tool in such balancing strategies; for instance, haptics can be used to increase the physical workload of a winning player and decrease the demand on a losing player.

Three main approaches are used for balancing players of different abilities: ladders and rankings, asymmetric roles, and dynamic difficulty adjustment. Many online games use ranking systems to group players of similar skill. This approach has the disadvantage of making it hard for friends and family to play together [33]. Another approach to balancing is to assign players different in-game roles. For example, in the Frozen Treasure Hunter exergame, one player uses an exercise bike to move an avatar, while a second player swings a Wii Remote to swat virtual snowballs [33].

Dynamic difficulty adjustment is a good fit with competitive games. This approach adjusts game parameters in real-time based on the player's performance [14]. Dynamic difficulty adjustment is used in the Age Invaders exergame to allow young and old players to play together [16]; parameters such as response time are dynamically adjusted based on the players' ages. The Heart Burn exergame and the Jogging over a Distance system provide dynamic difficulty adjustment by basing peoples' performance on their heart rate rather than their raw exercise power $[19,28]$. An advantage of dynamic difficulty adjustment is that people need not be aware that balancing is taking place.

We propose that haptics can complement existing approaches of using age or heart rate to dynamically adjust difficulty in exergames. The core idea is to use kinesthetic feedback to increase the game's difficulty for the stronger player. Sinclair et al. [27] have suggested a similar idea; but did not implement or test it. We illustrate this idea through the Truck Pull exergame, presented below. 


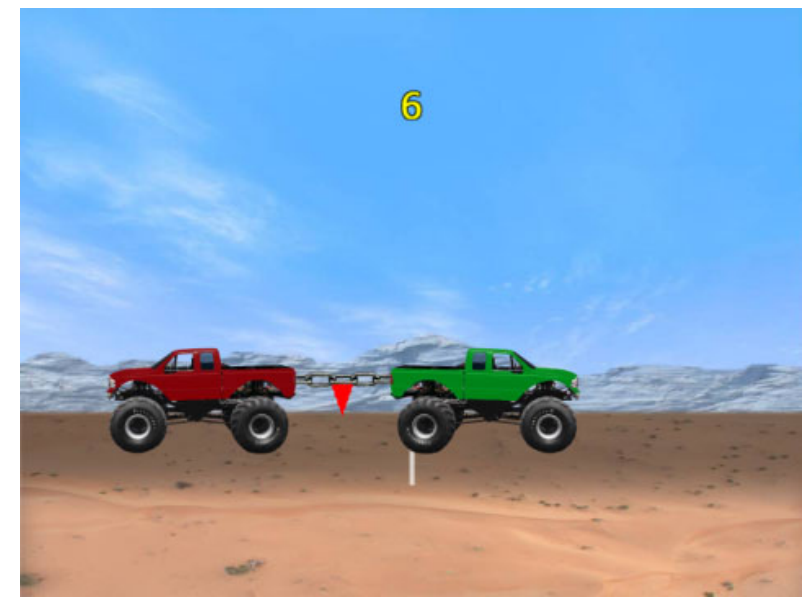

Fig. 1. The Truck Pull game. Player 1 (red) has taken the lead with 6 seconds remaining

Truck Pull: In Truck Pull, two players engage in a virtual tug-of-war by pedaling on their respective stationary bikes. Each player is represented by an on-screen truck. Both players' trucks are connected by a big chain (see figure 1). The trucks move in the direction of the player who is pedaling with the higher cadence. After one minute, the player who has moved the trucks closer to her side of the screen is the winner.

The pedal tension of a player's exercise bike increases as she moves the trucks to her side of the screen, and decreases as the trucks move to the opponent's side of the screen. This has the effect that the winning player must work significantly harder than the losing player. While the stronger person is still more likely to win, the haptic feedback keeps victories from becoming overly lopsided. As we shall see, the haptic version of Truck Pull leads to more balanced games, and players strongly prefer it to a non-haptic version.

\subsection{Guiding Players to Safe and Healthy Interaction}

Aerobic exercise is often prescribed at a specific intensity level, both to provide athletes with optimal training [30] and to avoid over-exertion in the less athletic [11]. Training in indoor cycling classes (or Spinning) is often based on maintaining a certain pedal cadence (e.g., 80 RPM). Runners often attempt to maintain a particular racing pace (e.g., 6 minutes $/ \mathrm{km}$ ). Taking a cue from ergometers in fitness studios, exergames can help users maintain a desired pace by providing displays showing cadence, heart rate or power output. Such displays risk focusing players on the physical activity rather than the game, possibly reducing immersion in the game's world and activities. We propose that haptic feedback can subtly guide exergame players to healthier and safer interaction without requiring immersion-breaking performance displays.

Current exergames do a poor job of guiding players towards appropriate levels of exertion. For instance, people playing Wii Sports do not achieve the exercise intensity recommended by the American College of Sports Medicine (ACSM) [12]. Alternatively, in more vigorous exergames, such as Heart Burn [28] or Swan Boat 
[1], it is possible for players to overexert themselves. In-game cues and dynamic difficulty adjustment have been proposed for regulating players' levels of exertion. For example, Ijsselsteijn et al. [15] developed an exergame with a virtual coach. The coach encourages a player to speed up if her heart rate is too low, or slow down if heart rate is too high. Similarly, exergames have been developed to adjust game difficulty based on a player's heart rate [6]. In this case, game difficulty increases if heart rate is low, or becomes easier as heart rate increases. While promising, the efficacy of these approaches have not been tested.

Haptic cues integrated into exercise equipment have been found to be effective at signaling people to adjust exercise intensity [10]. The use of haptic cues in exergames to guide players to effective levels of exercise has not been explored. In order to illustrate this approach, we created the Balloon Burst exergame, described below.

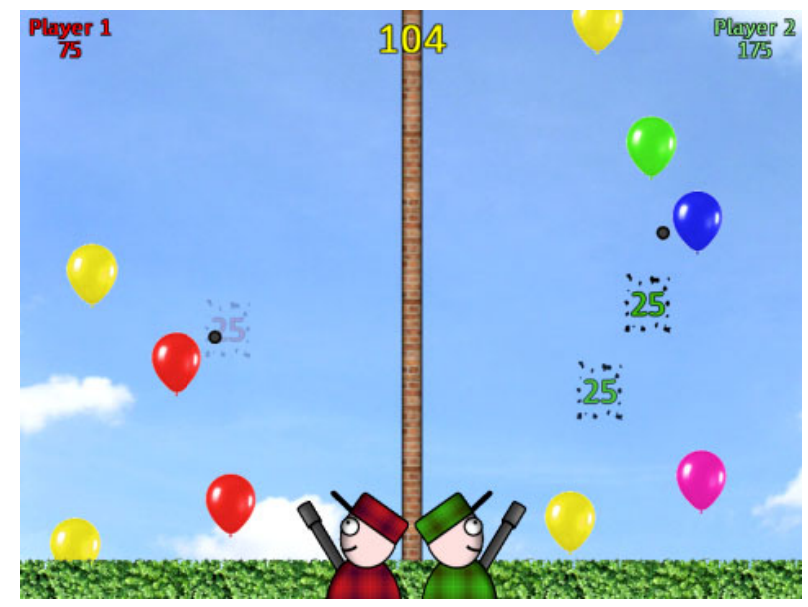

Fig. 2. The Balloon Burst game. Player 1 (red) has shot a balloon for 25 points; Player 2 (green) has just shot two balloons for 50 points; 104 seconds remain in the game.

Balloon Burst: In Balloon Burst, players attempt to shoot as many on-screen balloons as possible (see figure 2). A player is awarded 25 points for each balloon hit. At the end of two minutes, the player who has accumulated the most points is declared the winner. A wireless Xbox 360 gamepad and a recumbent exercise bike are used to interface with the game. Pressing the "A" button on the gamepad fires the player's virtual gun. The speed at which a player pedals on a stationary bike determines how often balloons are launched on screen. The faster the player pedals, the more frequently balloons are launched, and therefore the easier it is to score points.

In order to guide players to a safe level of exertion, a maximum pedal speed is set in the game. While the choice of maximum pedal speed is dependent on the user population and their exercise goals, for our experiment we chose an upper limit of 80 RPM based on recommendations for optimal bicycle training [5]. If a player exceeds this maximum pedal cadence, no balloons are launched. Balloons begin to launch again once pedal speed drops back below the maximum. It is therefore in the player's best interest to pedal as close to the maximum cadence as possible without exceeding it. Players receive tactile feedback indicating their cadence through the hand-held 
gamepad. The gamepad produces a pulsing sensation every time a balloon is launched; therefore, the faster a player pedals, the more rapidly the pulses occur. If the maximum pedal cadence is exceeded, the gamepad vibrates continuously, giving the player a cue to slow down.

In Balloon Burst, this haptic feedback allows players to focus on gameplay (i.e., trying to hit the balloons) rather than on a visual cadence monitor. The feedback is subtle, and therefore does not break the immersion of the game. Our experimental results will show that haptics are as effective as visual feedback for maintaining pedal cadence. However, the majority of players prefer the precision of visual feedback, and have trouble interpreting the gradations of haptic feedback.

\subsection{Increasing Presence}

Exergames aim to make exercise more enjoyable by shifting peoples' focus to gameplay, rather than the exertion of their physical activity. One way to achieve this goal is by increasing players' sense of presence in the game environment. Presence refers to the feeling of being in the virtual environment rather than the physical location [32]. Presence formalizes the more colloquial concept of "immersion" in virtual worlds.

Physical feedback has been shown to influence presence in virtual environments [24]. A lack of haptic feedback can decrease presence and task performance in collaborative groupware [25]. It has been proposed that force-feedback is important in exergames to simulate real-world activity. As we have discussed, several exergames do provide physical feedback to actions occurring on-screen. However, to our knowledge, the effect of haptic feedback on presence in exergames has not been experimentally investigated. Currently, it is unclear if the benefits of haptics in desktop environments [25] are transferable to exergames. To help fill this gap, we have created the Pedal Race exergame. In this game, haptic feedback is used to enhance the experience of riding over different types of virtual terrain.

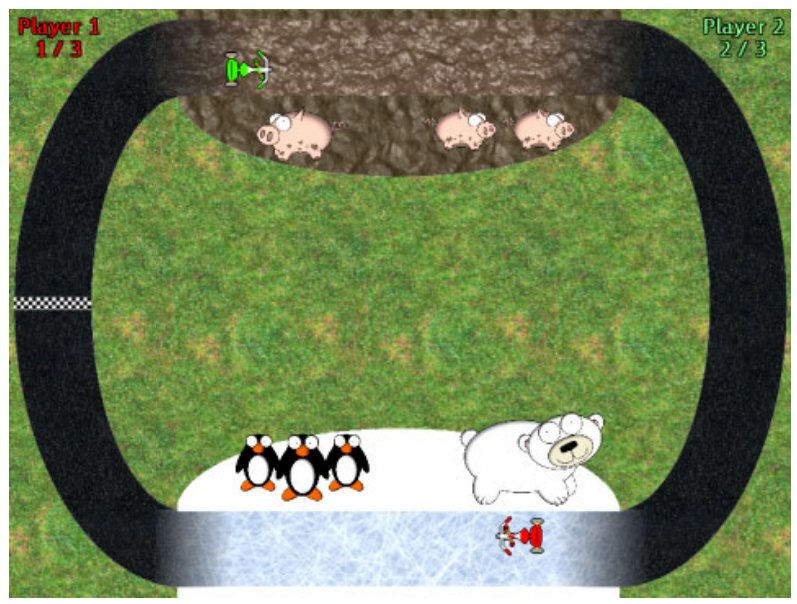

Fig. 3. The Pedal Race game. Player 1 (red trike) has completed 1 lap and is moving across the ice; Player 2 (green trike) has completed 2 laps and is in the mud. 
Pedal Race: In Pedal Race, players race virtual tricycles around a circular track. The first player to complete three laps wins the game. A player powers her tricycle by pedaling on a recumbent exercise bike. The faster a player pedals, the faster her tricycle will move. Steering of the tricycles is handled automatically by the game.

The virtual race track is made up of three types of terrain: asphalt, mud, and ice (see figure 3 ). The pedal tension of the exercise bike changes according the terrain; mud has twice as much tension as asphalt, and ice has half the tension.

The effect of this feedback is that when players enter the mud, they need to pedal harder to maintain the same speed. When they go over ice, the sudden reduction in tension evokes the feeling of spinning wheels. As we shall see, this haptic connection between the visual representation of terrain type and the change of pedal resistance leads to an increased sense of presence and increased enjoyment in the game.

\subsection{Summary}

In this section, we have discussed three ways that haptic feedback can enhance exergames. Although haptics have been included in exergames, empirical evidence supporting the benefits of haptic feedback is currently lacking. Through our three novel games, Truck Pull, Balloon Burst, and Pedal Race, we have shown how haptic feedback can be used to help balance games for people of disparate physical abilities, to help guide players to effective and healthy levels of exercise, and to enhance presence in exergames. In the next section, we discuss the results of our experimental investigation of the effectiveness of these techniques. These results illustrate advantages and pitfalls in the use of haptics in exergames.

\section{Evaluation}

We performed a user study to determine the effectiveness of haptic feedback in our Truck Pull, Balloon Burst, and Pedal Race games. The study was comprised of three experiments designed to explore how well these games balance group exercise, guide players to safe and healthy interaction, and increase virtual presence. Our study design is described below.

\subsection{Participants}

Twelve pairs of participants, 24 people in total, took part in the study. All participants were recruited from the university community. There were fifteen males and nine females, ranging in age from 18 to 42, with a mean age of 23. The majority of the participants reported playing video games at least a few times a month. Three people stated they performed exercise rarely or never, five stated they exercised once or twice per week, and 16 reported exercising at least three times a week. Most participating pairs had known each other for at least 2 months; however, 4 people did not know their partner prior to the study. Participants were chosen based on their ability to play video games using a gamepad, and to operate a recumbent exercise bicycle. We used the Physical Activity Readiness Questionnaire (PAR-Q) to screen participants whose health made it potentially inadvisable to perform exercise [26]. 


\subsection{Equipment}

The three exergames were designed as distributed two-player games. The games were developed in C\# using XNA 3.1. To interface with the games, players used a Tunturi E6R exercise bike attached to a Windows PC via a serial connection. In Balloon Burst, additional input was captured using a wireless Xbox 360 controller. The games were projected onto a large screen measuring 6' x 8' (see figure 4). Game music and sound effects were transmitted through 5.1 audio speakers. Two sets of equipment were used, allowing participants to be located in different rooms.

\subsection{Method}

Before completing the study trials, each participant was asked to complete a series of background questionnaires. Each pair of participants was then split into the two rooms containing the equipment described above. Participants played both a haptic and a non-haptic condition for each of the three exergames, for a total of six trials. The order of the exergames and the corresponding conditions were randomized. Prior to each trial, an experimenter described the game and condition to be completed. The game computer logged relevant game data such as pedal cadence and game score.

The study was designed to investigate the effectiveness of haptic feedback in exergames, not the long-term exercise efficacy of the games. Therefore, each trial was short, lasting between 1 and 5 minutes. Participants were given time between each trial to cool down and return to a resting state. Following the completion of all six trials, the two participants were brought back into one room for a post-experiment interview and debriefing.

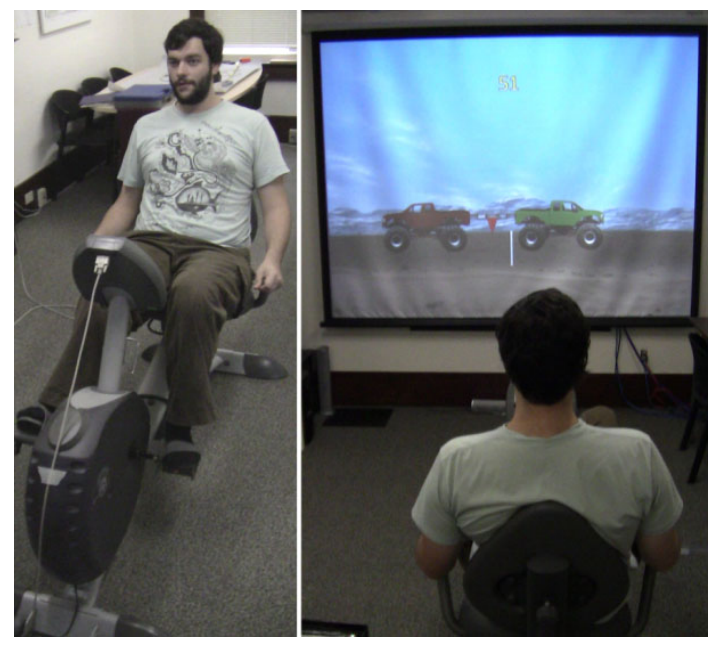

Fig. 4. Equipment setup. Left: player pedaling on bike; Right: player's view of the game

\section{Experiment 1: Balancing Group Exercise}

Although grouping can be a motivating factor for physical activity, it can be difficult for people of different capabilities to exercise together. We used Truck Pull to test our 
hypothesis that haptic feedback can help balance competition in exergames, and therefore enhance players' enjoyment. Kinesthetic feedback is a natural fit for balancing exergames specifically, since haptic force can exert more strain on peoples' muscles. The control version of Truck Pull was identical to the original, but where the haptic feedback was removed (i.e., pedal tension was constant).

\subsection{Experimental Method}

Half of the participants completed the haptic trial first, while the other half played the control version first. Each game of Truck Pull lasted for one minute. After completing both versions of the exergame, participants were asked how they perceived the balance of competition, and to state their preference between the versions.

The game was instrumented to record the position of the middle point between the players' trucks on one second intervals. This data allows game balance to be measured by the average distance (in pixels) of the center of the chain from the start line. A small average distance means that the game was well-balanced, with neither player maintaining a large advantage over the other.

\subsection{Results}

For each pair of participants, we compared average distances to the start line for each condition using a paired-samples t-test. Average distances to the start line were significantly lower in the haptic case $(M=39.98)$ than the control case $(M=66.63)$ at the alpha $=0.05$ level: $t(11)=2.41, p=0.035, d=0.98$. The reported Cohen's $d$ value is a measure of effect size [8]. The reported value of 0.98 indicates a large effect.

Participants were split between conditions when asked which version of Truck Pull gave them the best chance of winning (10/24 haptic, 9/24 control, and 5/24 no difference). However, a majority of participants felt the haptic version allowed for more equal competition (15/24 haptic, $7 / 24$ control, and 2/24 no difference), and was the preferred case overall (15/24 haptic, 5/24 control, and 4/24 no difference).

\subsection{Analysis}

In order to better support group exercise, exergames need to balance competition between players of disparate abilities. By adjusting the physical demands of the game based on a player's in-game performance, exergames can allow for more balanced group exercise. Balance in the Truck Pull exergame is measured by the average distance between the trucks to the starting line. Our results indicate that competition was significantly more balanced in the haptic version than in the control version. Additionally, a strong majority of participants stated the haptic version allowed for more equal competition than the control game. In Truck Pull, ultimately the stronger player is more likely to win. However, the haptics lead to closer competition, reducing the likelihood of "blow-out" victories, and leading to greater enjoyment.

When asked which version of Truck Pull they preferred, a strong majority of participants preferred the haptic case. In addition to the balancing effect, people found that haptic feedback added richness to the game. For instance, participants stated that the haptic version "requires more skill," "seemed to make the game more strategic," and "made the outcome more interesting." Two participants suggested that the haptic feedback added more realism to the game. One person said "it seemed more realistic for tension to change" and that the change in pedal tension "more simulated real life." 
The small minority of participants who favoured the control version of Truck Pull cited the lower physical effort required. Comments included that "pulling the opponent to the end was much easier" and "made it more worthwhile to pedal hard and take the lead because in the [haptic version] the lead is easily lost."

This experiment validates that haptic feedback is a useful tool for balancing exergames for people of disparate physical abilities.

\section{Experiment 2: Guiding Players to Safe and Healthy Interaction}

We used the Balloon Burst exergame to investigate the hypothesis that haptic cues can be effective at guiding players to safe and healthy levels of exercise. A control version of Balloon Burst was created in which the haptic representation of pedal cadence was removed, and cadence was instead displayed as a number on the screen. The games were instrumented to record cadence information at one-second intervals.

\subsection{Experimental Method}

Half of the participants played the haptic version first, and the other half started with the control version. Each game of Balloon Burst lasted for two minutes.

An engagement questionnaire [22] was used to measure the effects of the intensity cues on players' gameplay experience. (Questions pertaining to attention/flow and endurability were asked after each trial; questions on esthetics, novelty, and usability were completed after both versions of the game had been played.) After playing both versions, players were asked their preference between the versions, and which condition they believed allowed them to better maintain constant pedal cadence.

\subsection{Results}

To compare the effectiveness of the two forms of intensity cues at guiding players to desired exercise intensity, we compared the mean cadence for each condition using a paired-samples t-test. The mean cadence was slightly higher in the control case $(M=66.73)$ than in the haptic case $(M=66.06)$, but this difference was not significant at the alpha $=0.05$ level: $t(23)=0.41, p=0.69, d=0.06$. Post hoc analysis reveals that power was low (0.40).

Game scores for each version of Balloon Burst were compared. The mean scores were higher in the control case $(M=2,178.1)$ than the haptic version $(M=1,983.3)$. However, this difference was not significant at the alpha $=0.05$ level: $t(23)=1.650$, $p=0.113, d=0.4$. Post hoc analysis reveals that power was low (0.264).

Questionnaire results show that players preferred the control version of Balloon Burst (12/24 control, 8/24 haptic, and 4 no difference) and perceived that it allowed them to more easily maintain constant pedal cadence than the haptic game $(18 / 24$ control, 3/24 haptic, and 3/24 no difference). Scores from the engagement questionnaire were significantly higher for the control case $(M=193.50)$ than the haptic version $(M=184.20)$ at the alpha $=0.05$ level, $t(23)=4.76, p<0.001, d=0.33$.

\subsection{Analysis}

Questionnaire results show that players preferred the visual display of exercise intensity, felt it provided a more engaging experience, and allowed people to better 
maintain a constant pedal cadence than the haptic version. However, data logs from the game sessions demonstrate that there was no significant difference in pedal cadence or scores between the versions of Balloon Burst. Power was low for both the cadence and score comparisons, indicating the possibility of type 2 errors, but the standardized differences between the means were small enough that even had significance been obtained, the difference would be inconsequential to gameplay.

Player comments suggest that the visual display of exercise intensity was preferred due to the precision it offered. Participants stated that "seeing the numbers lets you know how close you are to 80RPM," and "with RPM displayed on screen, I can see if I have reached maximum RPM, therefore knowing my progress." Alternatively, some players found the haptic cues distracting because they had difficulty interpreting their meaning. Comments included that "the [haptic feedback] mapped to an otherwise unknown threshold. This was distracting and redundant rather than informative." and the visual cues were "less distracting from the [haptic feedback]... [which] allowed me to focus better."

In cases where participants preferred the haptic feedback to the visual feedback, people felt it allowed them to focus more on the game task. For instance, comments included "it was easier to focus on hitting the balloons [with the haptic feedback]," and "balloons were easier to keep track of [with haptic feedback]." This suggests a possible advantage of using the haptic channel to convey intensity information, freeing the visual channel for playing the game.

The central lesson from these findings is that people found it difficult to interpret the haptic cues, and therefore preferred the precise visual representation. Interestingly, the pedal cadence and score between the conditions were not significantly different, suggesting that the players' underrated their ability to interpret the haptic feedback. We believe that two issues contributed to players' dislike of the haptic interface. First, the haptic device itself was imprecise, making it difficult to distinguish between different levels of haptic intensity. Second, the linkage between cadence and feedback (vibration in the hands) was not obvious, leading to a disconnection between the virtual world and physical feedback.

\section{Experiment 3: Increasing Presence}

We used the Pedal Race exergame to test our hypothesis that haptic feedback can enhance peoples' sense of presence in a virtual game world. Presence is defined as "the subjective experience of being in one place or environment, even when one is physically situated in another" [32]. Specifically, we hypothesize that playing Pedal Race leads to higher presence than playing the non-haptic version of the game. In the non-haptic (control) version, players did not receive haptic feedback when changing terrain, but speed did change (double over ice, halved over mud.)

\subsection{Experimental Method}

Half of the participants played the haptic version of Pedal Race first, while the other half played the control version first. In each trial, players completed a total of three ingame laps, regardless of whether they won or lost. Overall, the races lasted an average of 89 seconds. 
An abbreviated version of the Presence Questionnaire (PQ) was used to measure peoples' sense of virtual presence [32]. This version of the PQ included questions most relevant to the study. Questions from the subscales of involvement and adaptation/immersion were selected for their focus on interaction and movement within a virtual environment. After completing each trial, participants completed the abbreviated PQ. Once both the haptic and control cases had been performed, participants were asked to compare the sense of realism between the versions, and express which version they preferred.

\subsection{Results}

To determine whether haptic feedback in exergames offers higher virtual presence than the non-haptic control case, we compared mean PQ responses for each condition using a paired-samples t-test. Mean scores were significantly higher in the haptic case $(M=5.02)$ than the control version $(M=4.53)$ at the alpha=0.05 level: $t(23)=3.25$ $p=0.004, d=0.44$.

When asked which version of Pedal Race felt more realistic, the majority of participants chose the haptic case: 21/24 chose haptic, 2/24 chose control, and 1/24 chose "no difference". The haptic version of Pedal Race was also selected as the most preferred by users (17/24 haptic, 4/24 control, and 3/24 no difference).

\subsection{Analysis}

The goal of exergames is to provide a captivating experience in order make exercise more palatable. It is therefore desirable to increase players' presence in the game's virtual world. We would expect that including haptic feedback to player's actions increases this sense of presence. Results from the PQ suggest that players' sense of presence was indeed higher in the haptic version of Pedal Race. This indicates that game designers should seriously consider haptics as a way of increasing players' involvement in the game.

Participants overwhelmingly responded that the haptic feedback made the game feel more realistic and preferred the haptic version of the game. These results indicate that it is possible to increase presence and realism in exergames by providing haptic feedback in response to in-game activity.

Post-experiment interviews indicated that most people preferred the haptic version of Pedal Race because of the improved realism it offered. Participants stated that the haptic game felt "how I imagine it would feel as you pedal through mud and ice" and that the "physics [of the exercise bike] made it feel more lifelike." In the few cases where players preferred the control version of Pedal Race, the tension-based feedback of the haptic game was seen as more physically challenging. One participant commented that the control case "put less strain physically than the version with the change in pedal tension." Another participant stated that "the change in tension made it more tiring" and "the [tension over the ice terrain] felt really weird because it was zero tension." Although a small minority did not like the increased effort required by the kinesthetic feedback, on balance it had a significantly positive impact on virtual presence. While this experiment involved a single game, it seems reasonable to assume that similar results would be found in other exergames where kinesthetic feedback can be used to enhance the primarily visual feedback presented by the game. 


\section{Discussion}

Each of our three exergames addresses an important problem in the design of exergames: balancing group exercise to allow people of different physical abilities to play together, guiding players to safe and healthy interaction, and providing an immersive experience to increase enjoyment of exercise. This indicates the diverse ways in which haptics can be applied to exergames. Because of the inherently physical nature of exergames, haptics are a natural fit, engaging a third sense in addition to traditional audio and visual feedback. These advantages make haptic feedback a valuable tool for exergame designers.

Players preferred the haptic version over the control version in two of our three games (Truck Pull, and Pedal Race). This suggests that the use of haptics increased the enjoyment of the games. Both use haptic feedback to report a physical phenomenon occurring in the game: in Truck Pull, pedal resistance is mapped to difficulty in pulling; in Pedal Race, modulating pedal tension allows players to feel variations in the virtual terrain. Questionnaire results from Pedal Race show that haptics improved peoples' sense of virtual presence. Similarly, players' comments indicate that people experienced greater presence in the haptic version of Truck Pull.

In contrast, the haptic feedback in Balloon Burst is less obviously associated with a physical phenomenon - players cannot apply their experience in the real world to explain how cadence should map to vibration of a hand-held controller. Although players were able to maintain appropriate pedal cadence, engagement scores and player feedback suggest that haptics did not enhance presence as intended. This hints that in order for haptics to improve virtual presence, the feedback should obviously correspond to what a player sees and hears in the game.

It is interesting to note that while haptic feedback was overwhelmingly preferred in Truck Pull and Pedal Race, this preference was not unanimous. These games provided kinesthetic feedback by adjusting pedal tension on the exercise bike, in some cases increasing the effort required from the player. Some participants highlighted this increase in physical difficulty as a negative, and reported preference for the nonhaptic versions of the games. This shows that resistance-based feedback should not be so drastic as to frustrate players with the amount of physical effort required to win.

\subsection{Limitations}

Haptic feedback in our three exergames was provided through an exercise bike or a hand-held gamepad. However, haptics may be difficult to implement in games where there is no physical contact with hardware. For instance, camera-based systems, such as the PlayStation Eye and Microsoft Kinect, do not currently offer a way of providing haptic feedback.

Truck Pull and Pedal Race used kinesthetic feedback (sensation of force and weight), and the Balloon Burst game utilized tactile feedback (sense of touch). The type of haptic feedback chosen may not be interchangeable. In Pedal Race, players receive feedback on the terrain they are crossing in the virtual environment through kinesthetic haptics. It would be difficult to represent more tactile sensations, such as a "bumpy" terrain, using resistance on the exercise bike. Similarly, in Truck Pull it is possible to change the physical effort required from players using pedal resistance. 
Achieving the same effect with tactile haptic feedback would be challenging. Alternatively, the Ballon Burst game allowed us to explore the application of tactile feedback to exergames. Several commercial active gaming devices do not support kinesthetic feedback, but have the ability to include tactile feedback. For example, the Wii Remote and the PlayStation Move accessory support rumble feedback.

\subsection{Design Issues}

The main lesson from this work is that designers should consider haptic feedback in exergames. Our exergames demonstrate that integrating haptics is feasible and can enhance interaction. The improved immersion seen in our third experiment, and the general preference for two of our three haptic games, provides support for increased use of haptic feedback in general. Our experience results in a set of issues for designers to consider:

- Haptics can serve diverse purposes in exergames. Haptic feedback can help to address several critical aspects in the design of exergames. Specifically, we demonstrated that haptics can improve balance in multiplayer exergames, help guide people to healthy and safe levels of interaction, and increase virtual presence. Designers should consider these, and possibly other, applications of haptic feedback in order to improve the richness of interactions in exergames.

- Haptic feedback should have a clear link to physical situations in the game environment. Our results from experiments with Truck Pull and Pedal Race show that haptics need to be consistent with physical actions occurring in the on-screen world. Designers should attempt to make logical mappings from observable events to haptic feedback in order in increase immersion. Our Balloon Burst game shows the pitfall of using overly abstract mappings that players find hard to interpret.

- Strategies for integrating haptics are limited by the capabilities of current hardware. Designers of exergames need to recognize the limitations in haptic feedback provided by existing gaming platforms. The Wii provides only tactile feedback through vibration in the Wii Remote controller. The otherwise excellent PCGamerBike Mini bicycle peripheral provides no programmatic control over its tension setting. Microsoft's Kinect platform is vision-based, and therefore can provide no haptic feedback at all. We encourage developers of active input peripherals to consider the importance of haptics in exergames. Even when haptic feedback is possible, it may not provide sufficient resolution for all purposes. As we saw, the Xbox gamepad has limited vibration settings. In Balloon Burst, we simulated intensity by pulsing the vibrations. As our experiment showed, players reported difficulty in distinguishing between these speeds of the Xbox gamepad. Designers should therefore perform user testing with haptic strategies in order to ensure that they work with all intended hardware platforms.

\subsection{Future Work}

All three of the exergames used in this work were based on exercise bikes. As the variety of exergaming peripherals increases, it will be important to support haptic feedback across different hardware. For instance, kinesthetic feedback could be achieved by changing the incline on a treadmill, or adjusting the resistance on a 
rowing machine. This will allow for better portability of haptic exergames across different platforms.

Allowing for customization of haptic feedback in exergames could improve its effectiveness. Physical strength varies from person to person, and therefore kinesthetic feedback may need to be tailored to each player. In Pedal Race, some people found the changes in tension not substantial enough, while others found it too drastic. Additionally, other forms of haptics could be investigated, such as feedback through temperature or air movement.

We were interested in investigating the effectiveness of haptic feedback in exergames, not the long-term health benefits of the games. In order to test our three hypotheses without exhausting the participants, each trial session was short. Further experiments would be interesting to measure players' view of haptic feedback over long-term play.

We have not yet explored the combination of our three applications of haptic feedback. In future, it will be important to examine whether haptics can simultaneously provide effective multiplayer balancing, guide people to healthy and safer interaction in exergames, and increase virtual presence.

\section{Conclusion}

Exergames currently lack the physicality of real-world sports and exercise. We propose that haptics - feedback related to peoples' sense of force and touch - can provide richer exergaming experiences. In our study, we found that haptic feedback can be applied to three areas relevant to exergames: supporting group exercise among players of different fitness levels, guiding people to safe and healthy interaction, and increasing players' sense of presence in game environments. Our experiments showed both advantages and pitfalls in applying haptics, leading to a set of principles to assist designers in applying haptic feedback to exergames.

Acknowledgements. We gratefully acknowledge Eril Berkok for his assistance in running the experiments. This work was supported by the Natural Science and Engineering Research Council of Canada, and the GRAND Network of Centers of Excellence.

\section{References}

1. Ahn, M., Kwon, S., Park, B., Cho, K., Choe, S.P., Hwang, I., Jang, H., Park, J., Rhee, Y., Song, J.: Running or Gaming. In: Proc. ACE, pp. 345-348 (2009)

2. Allender, S., Cowburn, G., Foster, C.: Understanding Participation in Sport and Physical Activity Among Children and Adults: a Review of Qualitative Studies. Health Education Research 21(6), 826-835 (2006)

3. Basdogan, C., Ho, C., Srinivasan, M.A., Slater, M.: An Experimental Study on the Role of Touch in Shared Virtual Environments. TOCHI 7(4), 443-460 (2000)

4. Beauchamp, M.R., Carron, A.V., McCutcheon, S., Harper, O.: Older Adults' Preferences for Exercising Alone Versus in Groups: Considering Contextual Congruence. Annals of Behavioral Medicine 33, 200-206 (2007) 
5. Brisswalter, J., Hausswirth, C., Smith, D., Vercruyssen, F., Vallier, J.M.: Energy Optimal Cadence vs. Freely-Chosen Cadence During Cycling: Effect of Exercise Duration. Int. J. Sports Medicine 22(1), 60-64 (2000)

6. Buttussi, F., Chittaro, L., Ranon, R., Verona, A.: Adaptation of graphics and gameplay in fitness games by exploiting motion and physiological sensors. In: Butz, A., Fisher, B., Krüger, A., Olivier, P., Owada, S. (eds.) SG 2007. LNCS, vol. 4569, pp. 85-96. Springer, Heidelberg (2007)

7. Chang, D.: Haptics: Gaming's New Sensation. Computer 35(8), 84-86 (2002)

8. Cohen, J.: Statistical Power Analysis for Behavioral Sciences, 2nd edn. Lawrence Erlbaum Associates, Mahwah (1988)

9. Faust, M., Yoo, Y.: Haptic Feedback in Pervasive Games. In: Proc. PerGames (2006)

10. Ferber, A., Peshkin, M., Colgate, J.E.: Using Kinesthetic and Tactile Cues to Maintain Exercise Intensity. IEEE Transactions on Haptics, 224-235 (2009)

11. Foster, C., Porcari, J.P., Battista, A., Udermann, B., Wright, G., Lucia, A.: The Risk in Exercise Training. American Journal of Lifestyle Medicine 2(4), 279-284 (2008)

12. Graves, L., Stratton, G., Ridgers, N.D., Cable, N.T.: Comparison of Energy Expenditure in Adolescents when Playing New Generation and Sedentary Computer Games: Cross Sectional Study. British Medical Journal 335, 1282-1284 (2007)

13. Hohepa, M., Schofield, G., Kolt, G.S.: Physical Activity: What do High School Students Think? Journal of Adolescent Health 39, 328-336 (2006)

14. Hunicke, R.: The Case for Dynamic Difficulty Adjustment in Games. In: Proc. ACE, pp. 429-433 (2005)

15. Ijsselsteijn, W., de Kort, Y., Westernik, J., de Jager, M., Bonants, R.: Fun and Sports: Enhancing the Home Fitness Experience. In: Rauterberg, M. (ed.) ICEC 2004. LNCS, vol. 3166, pp. 73-81. Springer, Heidelberg (2004)

16. Khoo, E.T., Lee, S.P., Cheok, A.D., Kodagoda, S., Zhou, Y., Toh, G.S.: Age Invaders: Social and Physical Inter-Generational Family Entertainment. In: Proc. CHI, pp. 243-246 (2006)

17. Mokka, S., Väätänen, A., Heinilä, J., Välkkynen, P.: Fitness Computer Game with a Bodily User Interface. In: Proc. ICEC, pp. 1-3 (2003)

18. Morelli, T., Foley, J., Columna, L., Lieberman, L., Folmer, E.: VI-Tennis: A Vibrotactile/Audio Exergame for Players who are Visually Impaired. In: Proc. FDG, pp. 147-154 (2010)

19. Mueller, F., Vetere, F., Gibbs, M.R., Agamanolis, S., Sheridan, J.: Jogging Over a Distance: The Influence of Design in Parallel Exertion Games. In: Proc. Siggraph (2010)

20. Mueller, F., Agamanolis, S., Gibbs, M., Vetere, F.: Remote Impact: Shadowboxing over a Distance. In: Proc. CHI, pp. 3531-3532 (2009)

21. Mueller, F., Stevens, G., Thorogood, A., O’Brien, S., Volker, W.: Sports Over a Distance. Personal and Ubiquitous Computing 11(8), 633-645 (2007)

22. O’Brien, H.L., Toms, E.G., Kelloway, E.K., Kelley, E.: Developing and Evaluating a Reliable Measure of User Engagement. In: Proc. American Society for Information Science and Technology, pp. 1-10 (2008)

23. Pollock, M., Gaesser, G., Butcher, J., Despres, J., Dishman, R., Franklin, B., Garber, C.: The Recommended Quantity and Quality of Exercise for Developing and Maintaining Cardiorespiratory and Muscular Fitness, and Flexibility in Healthy Adults. Med. \& Sci. in Sports and Exercise 30(6), 975-991 (1998)

24. Ramsamy, P., Haffegee, A., Jamieson, R., Alexandrov, V.: Using Haptics to Improve Immersion in Virtual Environments. In: Proc. ICCS, pp. 603-609 (2006) 
25. Sallnäs, E., Rassmus-Gröhn, K., Sjöström, C.: Supporting Presence in Collaborative Environments by Haptic Force Feedback. Trans. Comput. -Hum. Interact. 7(4), 461-476 (2000)

26. Shephard, R.J.: PAR-Q Canadian Home Fitness Test and Exercise Alternatives. Sports Medicine 5, 185-195 (1988)

27. Sinclair, J., Hingston, P., Masek, M., Nosaka, K.: Using a Virtual Body to Aid in Exergaming System Development. Comput. Graph. Appl. 29(2), 39-48 (2009)

28. Stach, T., Graham, T.C.N., Yim, J., Rhodes, R.E.: Heart Rate Control of Exercise Video Games. In: Proc. GI, pp. 125-132 (2009)

29. Suzuki, K., Jansson, H.: An Analysis of Driver's Steering Behaviour During Auditory or Haptic Warnings for the Designing of Lane Departure Warning System. JSAE Review 24(1), 65-70 (2003)

30. Swain, D.P., Franklin, B.A.: Comparison of Cardioprotective Benefits of Vigorous Versus Moderate Intensity Aerobic Exercise. The American Journal of Cardiology 97(1), 141147 (2006)

31. Warburton, D.E.R., Bredin, S.S.D., Horita, L.T.L., Zbogar, D., Scott, J.M., Esch, B.T.A., Rhodes, R.E.: The Health Benefits of Interactive Video Game Exercise. Applied Physiology, Nutrition, and Metabolism 32(3), 655-663 (2007)

32. Witmer, B.G., Singer, M.J.: Measuring Presence in Virtual Environments: A Presence Questionnaire. Presence: Teleoperators and Virtual Environments 7(3), 225-240 (1998)

33. Yim, J., Graham, T.C.N.: Using Games to Increase Exercise Motivation. In: Proc. Future Play, pp. 166-173 (2007) 